\title{
Web portals to revamp family practice, proponents say
}

A dvocates say Web platforms may revolutionize family medicine by allowing family physicians the ability to communicate with patients and provide medical services or advice online.

They also contend that by offering more comprehensive access to health information, the Web platforms empower patients to take more control of their health, and that online doctoring will save money for both patients and doctors. The former won't have to take time off work to see their doctor for a form or test result, while the latter will cut reception costs by utilizing online booking systems and automatic patient notification services.

When doctors sign up to one of the Web platforms - such as mypatientaccess.ca, HealthConnex, mydoctor.ca or myOSCAR - their patients get access, to varying degrees, to such information as medical records, lab results and longterm health indicators like blood pressure and blood-glucose levels. The platforms also allow patients to securely email their physicians.

But the services typically involve a fee, because provincial insurers do not generally remunerate doctors for the time they spend providing patients advice on the phone or Internet.

For some, such fees raise questions around equitable access to health care.

The fees essentially give patients the option of paying for more expedient and convenient access to a doctor, which could violate the Canada Health Act, says Mike McBane, national coordinator of the Canadian Health Coalition. "It's an area we're going to demand action on."

Patients who register with mypatientaccess.ca, for example, pay between $\$ 40$ and $\$ 75$ (the fee is set by the doctor) per email conversation with their physician.

Such fees offer more value than block fees (www.cmaj.ca/cgi/doi /10.1503/cmaj.109-3815) now being charged by many physicians, argues Moe Jiwan, president of Toronto,

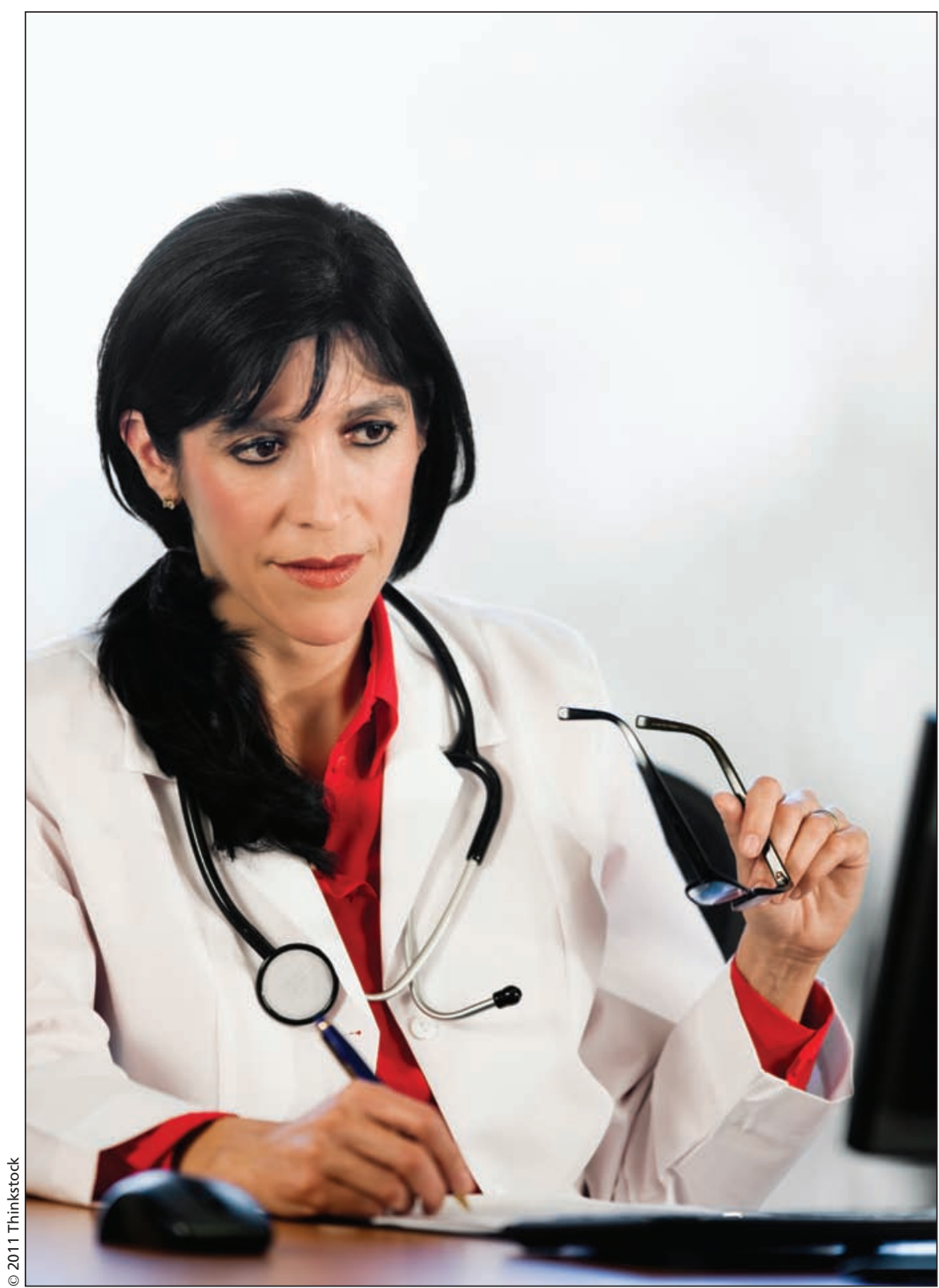

Patients who want to receive advice from their doctors via email will likely have to pay fees, which has raised questions of equitable access to health care.

Ontario-based Canadian Patient Access Inc., which partnered with Nightingale Informatix Corporation to create mypatientaccess.ca. "Our offering gives patients access to a suite of services never before brought forward and at a very reasonable rate," says Jiwan, who argues that the Web platforms promote greater accessibility for patients.
Jiwan also contends that econsults are rare and thus don't limit time available for in-office visits. But they are "helpful for patients who travel a lot. One of the physicians had a patient recently email him, 'Hey Doc, I'm in China and I'm finding that my sugar levels are all over the place. What should I do?"” 
Doctors who subscribe to my patientaccess.ca can use the portal for free, and basic services like online appointment scheduling are free for patients as well. Patients who want additional services, such as comprehensive lab reports and medical histories, pay $\$ 6.50$ or more per month. Separate fees are charged for econsults and online prescription refills. Jiwan says the bulk of the monies go to physicians, of whom about 3300 are using the portal, making it the most widely used platform now on the market.

At mydoctor.ca, which was launched in 2008 by the MD Physician Services, a division of the Canadian Medical Association, patients pay an annual fee of $\$ 19.95$. About 200 physicians have signed on, says Lyndon McPhail, product manager of MD Physician Services.
The portal is helpful for patients with chronic diseases because they can track indicators like blood sugar levels and weight over time, McPhail says.

Some family physicians give the portals a definite thumbs-up. Providing patients more ready access to lab results reduces their stress levels, says Dr. David Kaplan, associate family physician-in-chief at the North York General Hospital in Toronto. "Before, if we left a patient a message on Friday and told them to come to the office, all of a sudden they're anxious for the weekend. Now they can $\log$ in and access the results at any time."

But there are risks to online doctoring, acknowledges Dr. David Price, chair of Department of Family Medicine at McMaster University in Hamilton, Ontario, who will launch a univer- sity-developed portal for his family health team in September.

Price says that providing patients with access to diagnostic results or health indicators is "empowering," but some patients may misinterpret lab results that are uploaded onto the portal, for instance, and jump to the wrong conclusions.

While many believe that the portals will become more commonplace in the future and that physicians will likely be compensated by provincial governments for such services, the ramifications for health care remain murky. "I don't think we understand how this technology is going to change how we provide care and how we receive care as patients," Price says. — Wendy Glauser, Toronto, Ont.

CMAJ 2011. DOI:10.1503/cmaj.109-3915 